\title{
High-Dimensional White Matter Atlas Generation and Group Analysis
}

\author{
Lauren O'Donnell ${ }^{1,2}$ and Carl-Fredrik Westin ${ }^{1,3}$ \\ ${ }^{1}$ Computer Science and Artificial Intelligence Laboratory, \\ Massachusetts Institute of Technology, Cambridge MA, USA \\ lauren@csail.mit.edu \\ ${ }^{2}$ Harvard-MIT Division of Health Sciences and Technology, Cambridge MA, USA \\ ${ }^{3}$ Laboratory for Mathematics in Imaging, Brigham and Women's Hospital, \\ Harvard Medical School, Boston MA, USA*
}

\begin{abstract}
We present a two-step process including white matter atlas generation and automatic segmentation. Our atlas generation method is based on population fiber clustering. We produce an atlas which contains high-dimensional descriptors of fiber bundles as well as anatomical label information. We use the atlas to automatically segment tractography in the white matter of novel subjects and we present quantitative results (FA measurements) in segmented white matter regions from a small population. We demonstrate reproducibility of these measurements across scans. In addition, we introduce the idea of using clustering for automatic matching of anatomical structures across hemispheres.
\end{abstract}

\section{Introduction}

The use of diffusion MRI tractography [1 to select regions of interest (ROIs) in cerebral white matter has recently become a popular technique. The regions of interest, thought to correspond to particular anatomical white matter tracts, have been employed in quantitative analysis of scalar measures derived from the diffusion tensor, such as anisotropy values or mean diffusivities [234]. Ideally, in order to perform neuroscientific studies using white matter regions of interest, these regions should be automatically identified, correspond across subjects, and have anatomical labels. Our work addresses these three goals.

Related work on tractography segmentation includes multiple methods for calculation of tract-specific regions. One class of methods uses trajectories generated via tractography and groups them into regions either interactively or automatically. Manual interactive grouping of trajectories using multiple selection regions of interest (also known as "virtual dissection" [5]) has been performed

\footnotetext{
* We acknowledge the following grants: NIH NIBIB NAMIC U54-EB005149, NIH NCRR NAC P41-RR13218, NIH NCRR mBIRN U24-RR021382, and R01 MH 50747. Thank you to Susumu Mori at JHU for the diffusion MRI data (RO1 AG20012-01 / P41 RR15241-01A1). Thanks to Lilla Zollei for the congealing registration code and to Alexandra Golby, Tomas Izo, and Gordon Kindlmann for helpful conversations about this project.
} 
to create a fiber tract atlas [6] and in several clinical studies [234]. In fact, Partridge et al. found tractography-based definitions of a pyramidal tract ROI to be more reproducible than manual ROI drawing [4. Automated trajectory grouping using clustering algorithms has been proposed by Brun et al. [7, Gerig et al. 8], O'Donnell et al. [9, and Corouge et al. [10]. Automated trajectory grouping via atlas-based labeling of tractography was described by Maddah et al. 11] who manually created a tractography atlas and gave a method for transferring its labels to a novel subject. A second class of methods for generation of tract-specific ROIs (including approaches by Behrens et al. [12] and Parker et al. [13]) defines regions based on probabilistic connectivity measures.

We present a two-step method which automatically finds white matter structures present in a group of tractography datasets (atlas generation), and has a natural extension to find those structures in new datasets (automatic segmentation). The information that allows labeling of a new dataset is a high-dimensional representation of white matter structure that we call an atlas. However, it differs from traditional digital (voxel-based) atlases because it represents long-range connections from tractography rather than local voxel-scale information.

\section{Methods}

\subsection{Population Data}

Diffusion tensor MRI (single-shot spin echo EPI diffusion-weighted images, from a 1.5 T Philips scanner with SENSE parallel imaging, acquired along 30 noncollinear gradient directions $\left(b=700 \mathrm{~s} / \mathrm{mm}^{2}\right)$, with five non-diffusion-weighted T2 images, $2.5 \times 2.5 \mathrm{~mm} \times 2.5 \mathrm{~mm}$ voxels, and 50-60 slices per subject covering the entire hemispheres and the cerebellum) was analyzed for 15 subjects. Tractography was performed in each subject using Runge-Kutta order two integration, with the following parameters: seeding threshold of $c_{L} 0.25$, stopping threshold of $c_{L} 0.15$, step size $0.5 \mathrm{~mm}$, and minimum total length $25 \mathrm{~mm}$. The quantity $c_{L}$ is defined as $\frac{\lambda_{1}-\lambda_{2}}{\sqrt{\lambda_{1}^{2}+\lambda_{2}^{2}+\lambda_{3}^{2}}}$, where $\lambda_{1}, \lambda_{2}$, and $\lambda_{3}$ are the eigenvalues of the diffusion tensor sorted in descending order [14]. Group registration of subject FA (fractional anisotropy [15]) images was performed using the congealing algorithm [16] to calculate rotation, translation, and scaling (no shear terms). This registration was then applied to the trajectories generated via tractography.

\subsection{Step One: Atlas Generation}

To find common white matter structures in the population, we used our framework [9] for simultaneous clustering and matching of bundles across subjects. To complete the atlas, each cluster was given an expert anatomical label.

Similarity of Fiber Trajectories. Tractography clustering methods assume that trajectories that begin near each other, follow similar paths, and end near each other should belong to the same anatomical structure. Various affinity measures have been proposed in the literature to quantify this assumption 177810 . 
In this study we employed the mean closest point distance [10]18] which is defined as the average distance from each point on one trajectory to the nearest point on another trajectory. We symmetrized this distance by taking the minimum of the two possible distances. Each distance $\left(d_{i j}\right)$ was converted to an affinity $\left(a_{i j}\right)$ via a Gaussian kernel, $a_{i j}=e^{-} d_{i j}^{2} / \sigma^{2}$, with $\sigma$ of $30 \mathrm{~mm}$. The clustering and automatic segmentation is insensitive to small registration errors or anatomical differences due to the capture range of the similarity measure.

Similarity Across Hemispheres. To facilitate visual and quantitative comparison of anatomical structures which are present bilaterally, it is useful for the clusters (and their colors for visualization based on spectral embedding coordinates [17]) to correspond across hemispheres. To accomplish this goal, before computing the similarity metric we reflected one side of each brain across the midsagittal plane, such that trajectories with similar shapes and locations in either hemisphere would cluster together, automatically giving anatomical correspondences. (We defined the midsagittal plane as the midsagittal plane of the average group registered FA image.) This method gave better separation of some anatomical structures, for example the inferior parts of the cingulum from the inferior parts of the fornix. The improvement in clustering is due to the fact that reflecting across the midsagittal plane effectively doubles the number of prototype brain examples we have as input. Our atlas creation method is not dependent upon this reflection approach, however the bilateral matching is a useful additional property which we can obtain. We note that the success of the reflection approach would decrease in subjects with midline shift.

Clustering Algorithm. As in 9], to find common anatomical structures in the population using the calculated affinities, we performed Normalized Cuts spectral clustering [19]20, a method whose application to tractography was first proposed by Brun et al. 77. Spectral clustering methods employ eigenvectors of an affinity matrix to group data. We used the Nystrom method 20] to extend the eigenvector solution from a random subproblem (the normalized affinity matrix from a random sample of trajectories) to estimate a solution to the larger problem (eigenvectors for the whole population). The Nystrom method was used to estimate the leading eigenvectors of the normalized population affinity matrix

$$
\mathbf{D}^{-\frac{1}{2}} \mathbf{W} \mathbf{D}^{-\frac{1}{2}}
$$

where the symmetric matrix $\mathbf{W}$ contained affinities from all pairs of trajectories, and $\mathbf{D}$ was a diagonal matrix containing the row sums of $\mathbf{W}$. To estimate these eigenvectors, first a random sample of trajectories was compared to all other trajectories to generate a partial population affinity matrix with parts $\mathbf{A}$ (the square affinity matrix from the random subproblem) and $\mathbf{B}$ (the affinities between the random sample and all other trajectories). See Figure 1. The normalization of the $\mathbf{A}$ and $\mathbf{B}$ matrices by $\mathbf{D}$ was then estimated as [20]:

$$
\hat{\mathbf{d}}=\left[\begin{array}{c}
\mathbf{a}_{r}+\mathbf{b}_{r} \\
\mathbf{b}_{c}+\mathbf{B}^{T} \mathbf{A}^{-1} \mathbf{b}_{r}
\end{array}\right]
$$




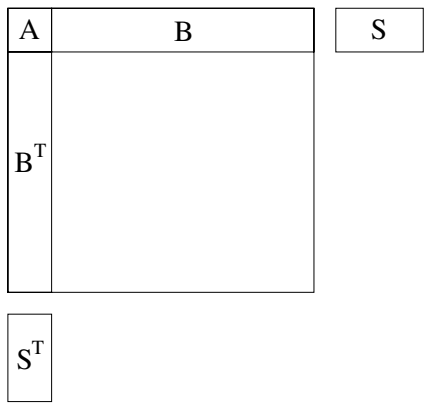

Fig. 1. Diagram of the parts of the affinity (tract similarity) matrix. $\mathbf{A}$ and $\mathbf{B}$ are used in atlas construction and $\mathbf{S}$ contains affinities for embedding a new subject.

where $\mathbf{a}_{r}$ and $\mathbf{b}_{r}$ are column vectors containing the row sums of $\mathbf{A}$ and $\mathbf{B}$, and $\mathbf{b}_{c}$ is the column sum of $\mathbf{B}$. The eigenvectors $\mathbf{U}$ and diagonal eigenvalue matrix $\boldsymbol{\Lambda}$ of the normalized matrix $\mathbf{A}$ were calculated, and finally the population eigenvectors $\overline{\mathbf{U}}$ were estimated via projection of normalized affinity values in $\mathbf{B}$ onto the eigenvector basis from A. $\overline{\mathbf{U}}$ was estimated via the following formula [20].

$$
\overline{\mathbf{U}}=\left[\begin{array}{c}
\mathbf{U} \\
\mathbf{B}^{T} \mathbf{U} \boldsymbol{\Lambda}^{-1}
\end{array}\right]
$$

Spectral embedding vectors were then calculated for each trajectory as $\mathbf{E}_{j}=$ $\frac{1}{\sqrt{\mathbf{D}_{j j}}}\left(\overline{\mathbf{U}}_{j, 2}, \overline{\mathbf{U}}_{j, 3}, \ldots, \overline{\mathbf{U}}_{j, n}\right)$. This generated a coordinate system, the spectral embedding space, where each trajectory was represented as a point, and similar trajectories were embedded near each other. K-means clustering was then performed on these embedding vectors, giving $k$ clusters.

Using Expert Knowledge to Label Atlas Clusters. This step introduced high-level anatomical knowledge to label the common white matter structures discovered by the clustering step. The $k$ clusters corresponded across subjects, in the sense that cluster number $i$ represented approximately the same region for each. So providing higher-level anatomical information was reduced to the problem of defining $k$ labels. We interactively labeled one subject, transferred the labels to the next, and worked through each atlas subject in this manner, ensuring that at the end all clusters had a high-level anatomical description. Due to the fact that tractography may cross from one anatomical structure to another, these anatomical labels represented the best approximate description of the regions discovered in atlas creation.

Atlas Contents. The atlas consisted of a set of cluster centroids in the embedding space, plus information for mapping new trajectories into this space. The necessary information was the random sample of input trajectories used in the affinity calculations, plus (as formulas 2 and 3 show) the basis vectors in $\mathbf{U} \boldsymbol{\Lambda}^{-1}$, the matrix $\mathbf{A}^{-1} \mathbf{b}_{r}$ for estimating the row sums, and the row (also column) sum $\mathbf{a}_{r}+\mathbf{b}_{r}$. In addition the atlas contained per-cluster anatomical labels. 


\subsection{Step Two: Automatic Segmentation}

By embedding new trajectories as points in the atlas embedding space, we were able to automatically segment tractography from novel subjects. First, affinity values were calculated by comparing the new subject's trajectories to the random sample of trajectories which was saved as part of the atlas. As shown in Figure 1, this generated a new partial affinity matrix $\mathbf{S}$, which then needed to be normalized using row and column sums as in (2). To keep the scaling of the old and new embedding vectors consistent, we estimated the row sum as

$$
\hat{\mathbf{d}}_{\text {row }}=\left[\mathbf{s}_{c}+\mathbf{S}^{T} \mathbf{A}^{-1} \mathbf{b}_{r}\right]
$$

where $\mathbf{s}_{c}$ is the column sum of $\mathbf{S}$. Then we employed the column sum from the original matrix.

$$
\hat{\mathbf{d}}_{c o l}=\left[\mathbf{a}_{r}+\mathbf{b}_{r}\right]
$$

Performing the scaling in this way made sense for two reasons. First, if we were to re-embed a trajectory that we had already seen (whose information was in $\mathbf{A}$ or $\mathbf{B}$ ) it would be mapped to the same location in the embedding space. Second, we would expect that each individual new trajectory would not significantly change the column sum $\hat{\mathbf{d}}_{\text {col }}$ of the (entire $\mathbf{W}$ ) matrix, due to the fact that 30,000 trajectories were used in creation of the original atlas affinity matrices. Thus the scaling applied to a novel trajectory was basically the same as that which would have been applied if it were part of the original clustering problem.

After normalization of the $\mathbf{S}$ matrix, the embedding vectors were calculated as in (3) for the $\mathbf{B}$ matrix. This step successfully embedded new trajectories as points in the clustered and labeled atlas embedding space. To create the automatic tractography segmentation, each new subject's trajectories were labeled according to the nearest cluster centroid.

\subsection{Parameters in Atlas Creation and Labeling}

To form the atlas, we used tractography from subjects 1 through 10 (of 15) for the clustering and anatomical labeling. 3,000 trajectories were randomly selected from each subject as input to the clustering, giving 30,000 total trajectories to cluster. The size of the random sample used to create the $\mathbf{A}$ matrix was 2,500 and $k=200$ clusters were generated. Finally, we labeled 10,000 trajectories from each of the 15 subjects using the atlas. These 10,000 trajectories were then used for measurement of FA and for creation of the images in this paper.

\subsection{Region-Based Measurements}

Any scalar invariant $\left(c_{L}, \mathrm{FA}\right.$, mode, trace, eigenvalues, etc.) or the tensors themselves can be measured in the white matter ROIs (individual clusters or anatomically-labeled regions) defined by the atlas. To illustrate the method we calculated the FA using tensors sampled at each point on the trajectories. Another possibility would be to label voxels according to the clusters (or fraction thereof) they contain, and to use the original tensors directly for FA calculation. 


\section{Results}

\subsection{Automatic Segmentation of Novel Subjects}

Five novel subjects (11-15) were labeled using the atlas, and Figure 2 shows tractography from three of these subjects. Note that the labeling is consistent across subjects, and that major anatomical structures such as the corpus callosum, arcuate fasciculus, uncinate fasciculus, etc. are found in each subject.
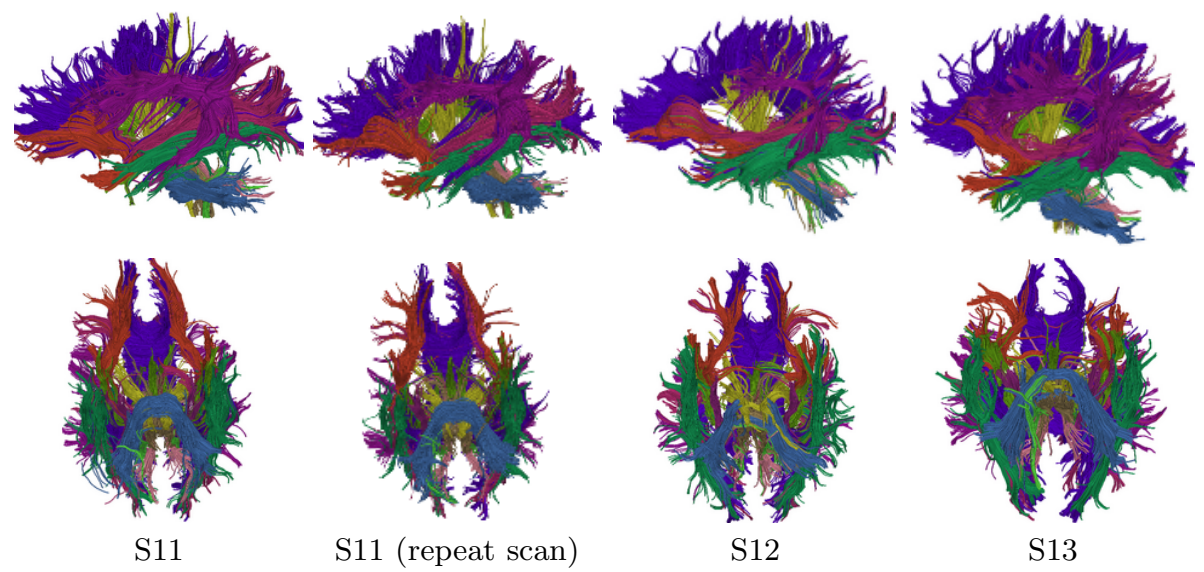

Fig. 2. Result of automatic segmentation of novel subjects (11-13). Selected regions are shown as follows: navy blue, corpus callosum; yellow, corticospinal fibers; purple, arcuate fasciculus/SLF region; orange, uncinate fasciculus; green, inferior longitudinal fasciculus; sky blue, middle cerebellar peduncle; light pink, superior cerebellar peduncle; hot pink, inferior occipitofrontal fasciculus.

\subsection{Measurement of Scalar Invariants in the Population}

Figure 3 shows the mean FA measured in selected anatomical regions in all subjects. The FA was measured bilaterally (both hemispheres together). The mean FA is relatively similar across subjects (in each structure) yet differs across structures, as would be expected from anatomically consistent group measurements. We have plotted using bar graphs in order to show the pattern across subjects where some were found to have consistently high or low FA relative to other subjects, as seen clearest in the corpus callosum and corona radiatae graphs.

\subsection{Reproducibility Experiment}

Using the atlas, we automatically segmented tractography and then measured FA in two subjects (10 and 11) who had each been scanned three times. Automatically segmented trajectories from two scans of subject 11 were shown in Figure 2 The quantitative measurements of FA demonstrate reproducibility and are shown in Figure 4 . 


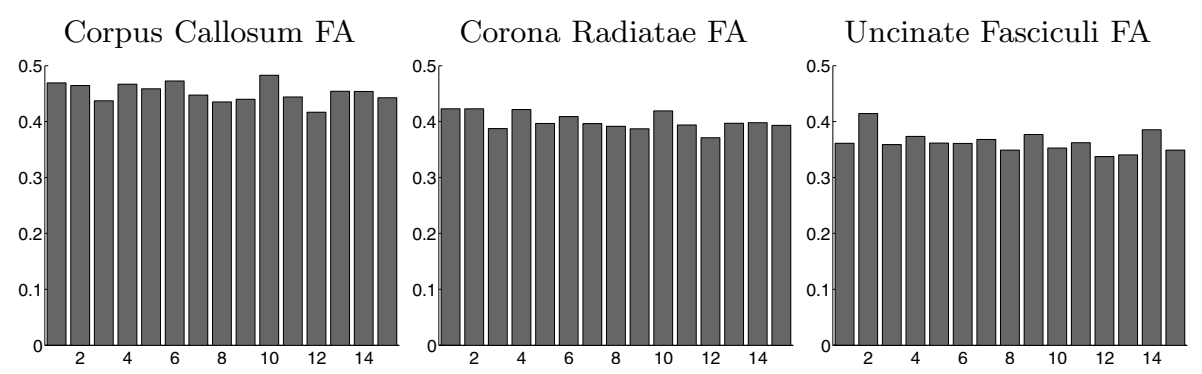

Fig. 3. Mean FA in three regions for 15 subjects. (The "corona radiatae" region includes trajectories in the cerebral peduncles, internal capsules, and corona radiatae.)

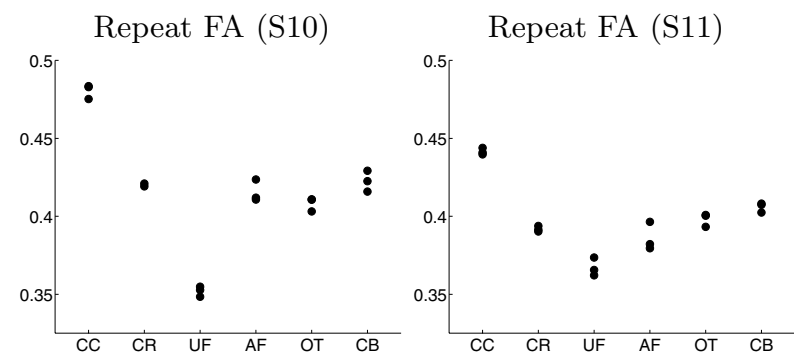

Fig. 4. Mean FA measurements from three repeat scans of two subjects (10 and 11). Abbreviations are: CC, corpus callosum; CR, corona radiatae (plus cerebral peduncles and internal capsules); UF, uncinate fasciculi; AF, arcuate fasciculi; OT, inferior longitudinal (occipitotemporal) fasciculi; and CB, cingulum bundles. The arcuate measurements were made in one bilateral cluster containing the traditional C-shaped fibers.

\section{Discussion and Conclusion}

An important point when labeling tractography with anatomical names is that the correspondence between tractography and anatomical regions is not always perfect, for instance one trajectory may traverse part of the arcuate fasciculus and part of the external capsule. An advantage of the clustering approach is that common such trajectories will form clusters, and can be labeled as a mix of structures. However, detection of uncommon trajectories merits investigation.

We have introduced a method for high-dimensional white matter atlas creation and automatic tractography segmentation which is robust to small errors in registration and can be used to find automatic region correspondences across hemispheres. In addition, we have shown that the tract-specific regions of interest obtained via automatic segmentation can be used to measure FA with consistency across subjects and reproducibility across scans of a single subject. Our technique enables quantitative neuroscience studies of the white matter in populations. 


\section{References}

1. Basser, P., Pajevic, S., Pierpaoli, C., Duda, J., Aldroubi, A.: In vivo fiber tractography using DT-MRI data. Magnetic Resonance in Medicine 44 (2000) 625-632

2. Concha, L., Beaulieu, C., Gross, D.W.: Bilateral limbic diffusion abnormalities in unilateral temporal lobe epilepsy. Annals of Neurology 57(2) (2004) 188 - 196

3. Jones, D.K., Catani, M., Pierpaoli, C., Reeves, S.J., Shergill, S.S., O'Sullivan, M., Golesworthy, P., McGuire, P., Horsfield, M.A., Simmons, A., Williams, S.C., Howard, R.J.: Age effects on diffusion tensor magnetic resonance imaging tractography measures of frontal cortex connections in schizophrenia. Human Brain Mapping (2005)

4. Partridge, S.C., Mukherjee, P., Berman, J.I., Henry, R.G., Miller, S.P., Lu, Y., Glenn, O.A., Ferriero, D.M., Barkovich, A.J., Vigneron, D.B.: Tractographybased quantitation of diffusion tensor imaging parameters in white matter tracts of preterm newborns. Magnetic Resonance in Medicine 22(4) (2005) 467-474

5. Catani, M., Howard, R.J., Pajevic, S., Jones, D.K.: Virtual in vivo interactive dissection of white matter fasciculi in the human brain. NeuroImage 17 (2002) $77-9$

6. Mori, S., Wakana, S., Nagae-Poetscher, L.M., van Zijl, P.C.: MRI Atlas of Human White Matter. Elsevier (2005)

7. Brun, A., Knutsson, H., Park, H.J., Shenton, M.E., Westin, C.F.: Clustering fiber traces using normalized cuts. In: MICCAI. (2004) 368-375

8. Gerig, G., Gouttard, S., Corouge, I.: Analysis of brain white matter via fiber tract modeling. In: EMBS. (2004) 426

9. O'Donnell, L., Westin, C.F.: White matter tract clustering and correspondence in populations. In: MICCAI. (2005)

10. Corouge, I., Gouttard, S., Gerig, G.: Towards a shape model of white matter fiber bundles using diffusion tensor MRI. In: ISBI. (2004) 344-347

11. Maddah, M., Mewes, A., Haker, S., Grimson, W.E.L., Warfield, S.: Automated atlas-based clustering of white matter fiber tracts from DTMRI. In: MICCAI. (2005) $188-195$

12. Behrens, T., Johansen-Berg, H., Woolrich, M., Smith, S., Wheeler-Kingshott, C., Boulby, P., Barker, G., Sillery, E., Sheehan, K., Ciccarelli, O., Thompson, A., Brady, J., Matthews, P.: Non-invasive mapping of connections between human thalamus and cortex using diffusion imaging. Nature Neuroscience 6 (2003) 750 757

13. Parker, G.J., Wheeler-Kingshott, C.A., Barker, G.J.: Estimating distributed anatomical connectivity using fast marching methods and diffusion tensor imaging. IEEE TMI 21(5) (2002) 505-512

14. Westin, C.F., Maier, S., Mamata, H., Nabavi, A., Jolesz, F., Kikinis, R.: Processing and visualization of diffusion tensor MRI. Medical Image Analysis 6(2) (2002) 93108

15. Basser, P., Pierpaoli, C.: Microstructural and physiological features of tissues elucidated by quantitative-diffusion-tensor MRI. J. Magn. Reson. Ser. B 111 (1996) 209-219

16. Zollei, L., Learned-Miller, E., Grimson, W.E.L., Wells III, W.M.: Efficient population registration of 3D data. In: ICCV 2005, Computer Vision for Biomedical Image Applications. (2005)

17. Brun, A., Park, H.J., Knutsson, H., Westin, C.F.: Coloring of DT-MRI fiber traces using Laplacian eigenmaps. In: EUROCAST. (2003) 564-572 
18. Wang, X., Tieu, K., Grimson, E.: Learning semantic scene models by trajectory analysis. Technical report, MIT CSAIL (2006)

19. Shi, J., Malik, J.: Normalized cuts and image segmentation. PAMI 22(8) (2000) 888-905

20. Fowlkes, C., Belongie, S., Chung, F., Malik, J.: Spectral grouping using the Nystrom method. PAMI 26(2) (2004) 214-225 